\title{
Intrinsic and Acquired Drug Resistance to LSD1 Inhibitors in SCLC Occurs through a TEAD4 Driven Transcriptional State
}

\section{Wen Yan}

Pfizer Global Research and Development

\section{Chi-Yeh Chung}

Pfizer Global Research and Development

\section{Tao Xie}

Pfizer Global Research and Development

\section{Mark Ozeck}

Pfizer Global Research and Development

\section{Timothy C. Nichols}

Pfizer Global Research and Development

Jessica Frey

Pfizer Global Research and Development

\section{Akshata R. Udyavar}

Arcus Biosciences

\section{Shikhar Sharma}

Pfizer Global Research and Development

Thomas Paul ( $\square$ Thomas.A.Paul@pfizer.com )

Pfizer Global Research and Development

\section{Research Article}

Keywords: LSD1, KDM1A, small cell lung cancer, epigenetic therapy, drug resistance

Posted Date: March 1st, 2021

DOI: https://doi.org/10.21203/rs.3.rs-249776/v1

License: (c) (i) This work is licensed under a Creative Commons Attribution 4.0 International License. Read Full License 


\section{Abstract}

\section{Background}

SCLC is a heterogeneous disease, consisting of intra-tumoral and inter-tumoral neuroendocrine (ASCL1/NEUROD1), mesenchymal-like, and YAP-driven transcriptional states. Lysine-specific demethylase 1 (LSD1/KDM1A) inhibitors have recently been progressed to clinical trials in small cell lung cancer (SCLC) based on a promising pre-clinical anti-tumor activity. Potential clinical limitation of LSD1 inhibitors are the heterogeneous drug responses that have been observed in SCLC cell lines and patientderived models. Based on these observations, we studied molecular and transcriptional signatures that predict patient response to this class of drugs.

Methods

LSD1 inhibitors, GSK690 and OG-86, were screened in a panel of SCLC cell line models facilitating the computational analysis of differentially expressed gene signatures correlated with intrinsic drug resistance, which were further evaluated experimentally in a SCLC mesenchymal variant form of NCl-H69 cells, NCl-H69V. Adaptive drug resistance was elucidated through bulk RNA-seq, ATAC-seq and single-cell RNA-seq, allowing study of transcriptional reprogramming in response to the drug treatment.

Results

Employing SCLC patient-derived transcriptional signatures, we define that SCLC cell lines sensitive to LSD1 inhibitors are enriched in neuroendocrine transcriptional markers while cell lines enriched in a mesenchymal-like transcriptional program demonstrate intrinsic resistance to LSD1 inhibitors. We have identified a reversible, adaptive resistance mechanism to LSD1 inhibitors through epigenetic reprogramming to a TEAD4-driven mesenchymal-like state.

\section{Conclusions}

Our data suggests that only a segment of SCLC patients, with a defined neuroendocrine differentiation state, will likely benefit from LSD1 inhibitors. It provides novel evidence for the selection of a TEAD4driven mesenchymal-like subpopulation resistant to LSD1 inhibitors in SCLC patients that may require effective drug combinations to sustain effective clinical responses.

\section{Background}

Small cell lung cancer (SCLC) is a neuroendocrine carcinoma that exhibits aggressive malignancy and a high propensity for early metastasis. In addition to common mutations in RB1 and TP53, molecular profiling studies have identified an epigenetic-basis for SCLC disease initiation and progression featuring frequent mutations in chromatin modifying genes including CREBBP, EP300 and MLL1/2 as well as alterations in the distribution of DNA methylation [1]. Based on these observations, epigenetic drugs have been explored for therapeutic intervention to target tumor-specific epigenetic vulnerabilities and reverse 
acquired epigenetic changes driving tumor growth and survival. Promising in vitro and in vivo preclinical data led to clinical trials exploring the use of histone deacetylase inhibitors including romidepsin or panobinostat in relapsed SCLC. Although both agents were well-tolerated and demonstrated some evidence of tumor shrinkage and sustained stable disease both clinical studies failed to demonstrate a significant benefit warranting continued development [2].

The emergence of a new generation of epigenetic drugs targeting specific transcriptional regulators of SCLC oncogenesis including BRD4, LSD1, and EZH2, has renewed interest in exploring epigenetic therapies in SCLC [3-5]. Among this class of new epigenetic drugs, inhibitors of the histone lysine demethylase LSD1 (KDM1A) have gained significant attention [6]. LSD1 is a member of the BRAF-HDAC (BHC) co-repressor complex shown to regulate expression of neuronal gene programs through interaction with REST [7-12]. In addition to the previously reported anti-tumor activity in acute myeloid leukemia, LSD1 inhibitors have shown lineage-specific activity in SCLC cell lines and patient-derived xenograft models $[5,6,13-15]$. Although the mechanism of growth arrest conferred by these drugs is unclear, LSD1 has been shown to regulate neuroendocrine transcriptional programs critical for SCLC growth $[5,13]$. Several recent reports have suggested that the primary mechanism of action of LSD1 inhibitors in both AML and SCLC occurs not only through catalytic inhibition of LSD1 enzymatic function but also through inhibition of the interaction of LSD1 with SNAG-domain transcriptional factors such as INSM and GFI1B $[14,15]$. Through these inhibitory mechanisms, LSD1 inhibitors can impact the expression of neuroendocrine lineage-specific regulators such as ASCL1, required for SCLC lineage specification.

Recent studies have characterized SCLC as heterogeneous population, consisting of intra-tumoral and inter-tumoral neuroendocrine (ASCL1/NEUROD1), mesenchymal-like, and YAP-driven transcriptional states and subtypes $[16,17]$. The particular SCLC subtypes may define vulnerabilities to therapeutic targets. In a breadth of efficacy screen, Mohammad et al. identified only a subset of SCLC cell lines and primary samples with a DNA hypomethylation signature undergoing growth inhibition in response to GSK2879552 [5]. Similarly, in a panel of SCLC PDX tumors, Augert et al. identified selective activity of ORY-1001 only in models capable of NOTCH pathway activation [13]. Moreover, many sensitive SCLC cell lines and primary tumor models display only partial responses to LSD1 inhibition even after long durations of treatment. The recent advancement of both irreversible and reversible LSD1 inhibitors into SCLC clinical trials warrants the study of potential drug resistance mechanisms that may prevent patients from responding to this class of drugs.

In this study, we have addressed potential intrinsic and acquired drug resistance mechanisms to LSD1 inhibitors in SCLC. Utilizing a recently identified gene co-expression network defining SCLC neuroendocrine and mesenchymal states $[18,19]$ we have identified that sensitivity to LSD1 inhibitors in SCLC is confined primarily to cell lines that express neuroendocrine transcriptional programs. Heterogeneous drug responses in SCLC cell lines reflect a pre-existing cell intrinsic drug resistance mechanism enriched in cell lines expressing mesenchymal-like transcriptional programs. Using single cell RNA-seq and ATAC-seq we demonstrate that continuous treatment with LSD1 inhibitors results in the emergence of drug tolerant subclones with a de novo mesenchymal-like transcriptional state driven 
through a TEAD4 transcription factor program. We also highlight that acquired resistance to LSD1 inhibitors appears as an "epi-stable" state. Under drug withdrawal, drug tolerant SCLC cells transition between mesenchymal and neuroendocrine phenotypes and regain sensitivity to the drug. Collectively our data provide novel insight in the mechanism contributing to heterogeneous responsiveness of SCLC to LSD1 inhibitors and selection of mesenchymal-like enriched subclones are likely to present a barrier to effective single-agent responses in the clinic.

\section{Materials And Methods}

\section{Cell lines and reagents}

Human SCLC cell lines used in this study were obtained from the American Type Culture Collection (ATCC), Sigma-Aldrich, or Deutsche Sammlung von Mikroorganismen und Zellkulturen (DSMZ) and grown in manufacturer's specified growth medium and environmental conditions. Cell growth medium was purchased from Life Technologies or Lonza.

GSK690 [20] and OG-86 [21] were synthesized by WuXi AppTec using previously disclosed structures. Structures were confirmed using NMR (Nuclear magnetic resonance spectroscopy) and LCMS (Liquid chromatography-mass spectrometry). Compounds were solvated in DMSO (Sigma) at 30mM for use in in vitro experiments.

\section{Preparation of cell extracts and western blot analysis}

Adherent and suspension cell populations were collected and washed in PBS prior to lysis in RIPA buffer (Sigma) with protease inhibitor cocktails (Roche) plus PMSF. Cell lysates were briefly sonicated prior and pre-cleared by centrifugation. $30 \mu \mathrm{g}$ of protein was loaded on $4-12 \%$ SDS-PAGE gels and transferred to nitrocellulose membranes. Samples were incubated overnight at $4^{\circ} \mathrm{C}$ in primary antibodies and $1 \mathrm{hr}$ at room temperature in secondary antibodies. Imaging was performed using LiCor imaging system.

Antibodies used in this study are KDM1A (Millipore cs207350, Bethyl A300-215A), NSE (CST 9536), SYP (CST 12270), GRP (Sigma HPA007314), NCAM (CST 3576), CHGA (Abcam 45179), FOXA2 (CST 3143), OVOL2 (Abcam 129161), LEF1 (CST 2286), ASCL1 (Abnova H0429-M02), SOX2 (CST 4900), INSM1 (Abcam 170876), NEUROD1 (117562), SMAD3 (CST 9513), NFKB2 (CST 3017), CMYC (CST 9402), MITF (Abcam 140606), SNAIL (CST 3895), VIM (CST 5741), CDH2 (CST 14215), CDH1 (CST 3195P), ZEB1 (CST 3396P)

\section{Long-term cell proliferation assays}

For drug sensitivity studies, cell lines were plated in triplicate $10 \mathrm{~cm}^{2}$ dishes at drug concentrations of $1000,300,100,30,10,3$ and 1 nM in DMSO. Every 3 or 4 days, adherent and suspension cells were collected and $2 \mathrm{ml}$ of cells was transferred to a new $10 \mathrm{~cm}^{2}$ dishes for subsequent culture. $8 \mathrm{ml}$ of fresh cell culture medium was added to each plate and drug was added at proper concentrations to each dish. Three aliquots of $1 \mathrm{ml}$ of cells was collected in $1.5 \mathrm{ml}$ microcentrifuge tube, concentrated by 
centrifugation to a $100 \mu \mathrm{l}$ volume and added to a 96 well plate. Relative cell numbers were determined either by cell counting of three independent aliquots using a hemocytometer or by CellTiter-Glo reagent (Promega).

\section{Tissue Microarray}

Lung cancer tumor microarray (14-140) was purchased from US Biomax, Inc. Immunohistochemical staining and evaluation was performed on a single TMA slide containing 200 microarray dot tissue samples representing a broad array of human lung cancer histologic subtypes as well the inclusion of 20 normal lung tissue samples for comparison. Immunohistochemical procedure and staining was developed and optimized with known LSD1 positive control tissues to ensure proper specificity. Pathologist scoring method for all evaluated TMA cores was semi-quantitative and based on a range of tumor cell positivity corresponding to each score, with a score of 1 indicating rare positivity, 2 showing 3$10 \%$ positivity, 3 showing $11-30 \%$ positivity and 4 for everything greater than 30 .

Immunohistochemical stained slides were prepared using a Leica Bond automated staining system. Briefly, slides underwent antigen retrieval (Leica ER2) for 20 minutes followed by incubation with rabbit anti-LSD1 1:100 (Cell Signaling Technology \#2139) for 40 minutes at room temperature. Bond Polymer Refine (Bond \#DS9800) kit with Poly-HRP and 3,3-Diaminobenzidine tetrahyrochloride (DAB substrate chromogen) was used to detect and visualize the LSD1 staining. Finally, the slides were counterstained with Hematoxylin, dehydrated and mounted.

\section{RNA-seq and expression profiling experiments}

RNA-seq was accomplished on SCLC cell lines treated in triplicate with DMSO or $0.3 \mu \mathrm{M}$ GSK690 for either 3 days or 10 days. RNAseq libraries were generated following manufacture's protocols and sequenced paired-end on the HiSeq 2000 at read length 50nt or 100nt (only for COR-L88). FastQ data generated by HiSeq was QCed and trimmed before being aligned to the HG19 human reference genome and then quantified using the RSEM package [22] The RNAseq DE (Differential expression) analysis was done by using the DESeq R package after RSEM [23]. For this analysis, the program used raw counts as input and ran its normalization method using a scaling factor computed as the median of the ratio, for each gene, of its read count over its geometric mean across all lanes.

The SCLC cell line mRNA expression data was downloaded from CCLE 19Q1 [24]. For differential expression analysis, genes that are not expressed (TPM $<3$ in all cell lines) are first removed, and moderated t test was conducted using Limma package with Empirical Bayes shrinkage [25] in sensitive $(n=10)$ vs resistant $(n=12)$ cell lines using log2-TPM values with FDR multiple testing correction. Differential expression cutoff is set at FDR $<5 \%$ and $\mathrm{FC}>2$. The heatmaps were generated using the Complex Heatmap package in R [26]. To identify enriched pathways associated with LSD1 sensitivity, we calculated a significance score defined as: - $\log 2($ FDR) X (difference in mean TPM between sensitive and resistant lines) and loaded this score into GSEA pre-rank for pathway analysis [27]. The neuroendocrine $(\mathrm{NE})$ and mesenchymal-like $(\mathrm{ML})$ network signature genes for 53 SCLC CCLE cell lines were defined as previously described $[19,28]$. For overlapping analysis between differential genes and NE/ML network 
genes, hypergeometric test was performed between each gene set, and the median TPM value of NE or ML module genes were plotted with boxplot with two-sample t test on sensitive and resistant cell lines.

For TCGA SCLC tumors gene expression analysis, the 2015 UCologne RNA-seq z score data was downloaded from cBio portal (https://www.cbioportal.org/). Genes containing NA readings were removed, and duplicated gene names were merged by their mean expression $z$ score. This resulted in a total of 18598 genes in 81 patient tumors. To calculate gene signature scores, the median expression z score from each signature was calculated for each tumor. The gene expression heatmap was generated using the Complex Heatmap package in R [26].

\section{Single cell isolation and scRNA-seq}

$\mathrm{NCl}-\mathrm{H} 69$ cells were treated in duplicate with DMSO or $0.3 \mu \mathrm{M}$ GSK690 for 21 days. The single cell suspension was isolated by passing the harvested cells through a $40-\mu \mathrm{m}$ Flowmi $^{\text {im }}$ tip strainer followed by incubation with $0.25 \%$ Trypsin-EDTA in ice for $10 \mathrm{~min}$ and at $37^{\circ} \mathrm{C}$ for $10 \mathrm{~min}$ to get a final concentration of $3 \times 10^{5}$ cells $/ \mathrm{ml}$. The single cells were then processed through the Chromium $3^{\prime}$ Single Cell Platform using v2 reagents (10X Genomics, Pleasanton) per the manufacturer's protocol. In brief, a total of 6536 single cells were detected with 3603 cells analyzed in the DMSO vehicle group and 2903 in LSD1 inhibitor-treated group. The cells were partitioned into Gel Beads in Emulsion in the Chromium instrument, where cell lysis and barcoded reverse transcription of RNA occurred, followed by amplification, shearing and $5^{\prime}$ adaptor and sample index attachment. Libraries were sequenced on an Illumina HiSeq 4000 .

For single cell RNA-seq analysis, gene-cell count matrix was generated with Cellranger pipeline (10X Genomics). All downstream analyses, including cell filtering, data normalization and cell clustering were performed using the R Seurat V3 package [29]. In brief, to remove low quality data, cells that have unique gene counts between 200-6000 and mitochondrial reads $<10 \%$ were kept for further analysis. Read counts were then normalized for total counts in each cell, scaled to 10000 , and then log transformed. To remove confounding factors associated with assay quality and cell cycle stages, we used SCTransform [30] to regress out percent mitochondrial reads, percent ribosomal reads, and cell cycle S/G2M score in each single cell. Top 3000 variable genes were selected for downstream analysis. To facilitate single cell comparison between control and LSD1 inhibitor treatment, we used Harmony package [31] with default settings to integrate single cell data, and used UMAP [32] for two dimensional data embedding.

Differentially expressed genes between each cluster or control vs drug treated cells were called using the Seurat "FindMarkers" function with these parameters: $\min . p c t=0.2$, logfc. threshold $=0.25$, with a cutoff of $\mathrm{FDR}<5 \%$. Gene expression values or scores in UMAP were capped at $5 \%$ and $95 \%$ quantile to eliminate outlier bias during data representation.

\section{ATAC-seq and Data Analysis}

$\mathrm{NCl}-\mathrm{H} 69$ cells were treated in triplicate with DMSO or $0.3 \mu \mathrm{M}$ GSK690 for 21 days. The transposition assay was performed. Briefly, 50,000 nuclei from each sample were used in each reaction with $20 \mu \mathrm{l}$ of transposition mix and incubated at $37^{\circ} \mathrm{C}$ for 60 minutes. qPCR was performed and 8 cycles were 
performed. The library was purified with Ampure XP beads (A63880, Beckman Coulter), quantified using Qubit (Qubit dsDNA HS Assay Kit, Q32851, Thermo Fisher Scientific) and checked for size distribution using 4200 TapeStation (High Sensitivity D1000 ScreenTape, 5067-5584, Agilent Technologies). Sequencing was performed with Illumina HiSeq4000 (50 bp PE, Nextera v2 libraries).

For ATAC-seq data analysis, paired-end sequencing reads for each sample were mapped to hg38 human reference genome with Bowtie2 [33] using the following parameters: - $t$-X 2000 -no-mixed -no-discordant. Sam files were converted into Bam with Samtools. Peak calling was performed with MACS2 [34] using these parameters: callpeak -g hs -nolambda -nomodel -shift - 100 --extsize 200 -bdg -SPMR -q 5e-2. Peaks overlap with ENCODE black list regions were removed from downstream analysis using Bedtools. Bedgraph files were converted to Bigwig using bedGraphToBigWig [35] and signal tracks were visualized with IGV [36]. To correct for ATAC-seq bias, we extracted genome wide signal at 100 bp resolution using DeepTools [37], and performed quantile normalization on the signal data using the R package preprocessCore (https://rdrr.io/bioc/preprocessCore/). Differential peak calling between the control and drug treated samples was performed with DESeq2 [38], using merged peaks between all samples (total 250646 peaks). Significant differential peaks were defined as: FDR $<0.01 \&$ fold change $>2.5$, and the mean read count for each peak is $>40 \%$ quantile of all mean read count (to remove low signal regions). This results in 548 open peaks and 838 closed peaks after drug treatment. GREAT analysis [39] was performed on the differential peaks to identify pathways associated with the drug treatment. To predict transcription factor (TF) binding perturbed by the drug treatment, we employed chromVar package [40] with the "human_pwms_v2 motif" database to identify TF binging DNA motifs that are significantly open or closed upon treatment. T test with FDR correction was performed on all TF z scores to identify significant TF motifs.

\section{Results}

\section{LSD1 is highly expressed in small cell lung cancer relative to other lung cancer subtypes}

Over-expression of chromatin modifiers has been shown to influence their function in cancer [41]. In lung cancer, LSD1 mRNA expression is highly expressed in SCLC relative to other lung cancer cell lines

(Fig. 1A). Expression datasets from Clinical Lung Cancer Genome Project (CLCGP) [42] confirms LSD1 is also expressed highly relative to other lung cancer subtypes in primary patient samples (Fig. 1B). To further confirm LSD1 expression levels at the protein level, we conducted immunohistochemistry $(\mathrm{IHC})$ on a lung cancer tissue microarray. IHC staining confirmed a statistically significantly higher staining intensity in SCLC specimens relative to that of squamous cell carcinoma, adenocarcinoma, and large cell carcinoma (1-way ANOVA Fig. 1C, 1D).

\section{SCLC cell lines show heterogeneous responses to LSD1 inhibitors}


Resulting from the observation of high expression of LSD1 in SCLC, we assessed whether SCLC cell lines were dependent on LSD1 activity for their growth. Using two shRNAs targeting LSD1 we observed growth inhibition in the $\mathrm{NCl}-\mathrm{H} 526$ cell line that became strongest after 14 days of target knockdown (Figure S1A). Interestingly, DMS-114 cells did not appear sensitive to LSD1 knockdown over 14 days even though there was a significant loss of LSD1 protein level by western blot (Figure S1B).

Several reports have recently described sensitivity in SCLC cell lines to LSD1 inhibitors. To study this further, we profiled drug sensitivity to the FAD reversible inhibitor GSK690 $(\mathrm{Ki}=4 \mathrm{nM})[20]$ as well as the FAD irreversible compound OG-86 (IC50 $=47 \mathrm{nM})$ [21] in a panel of SCLC cell lines (Figure S2). Due to the delayed onset of proliferation changes observed in shRNA experiments we tested the effects of GSK690 and OG-86 on SCLC cell line growth in long-term proliferation assays of 17-21 days. Consistent with shRNA results, we observed differential responses in many SCLC cell lines. In NCl-H1417, NCl-H187, NClH889 cells, dose-dependent growth inhibition was initially observed at 7-to-10 days of both GSK690 and OG-86 treatment with continued effects observed until day 17-21 (Figure S3A-C). DMS-114 cells, which were insensitive to LSD1 shRNA knockdown, were also insensitive to both GSK690 and OG-86 up to 17

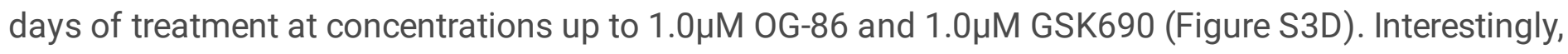
the anti-proliferative effects of LSD1 inhibitors plateau in their percent inhibition even after 17-21 days of continuous drug treatment due to the emergence of a subpopulation of slowly proliferative persister cells in $\mathrm{NCl}-\mathrm{H} 1417$ and $\mathrm{NCl}-\mathrm{H} 187$ cells (Figure S3). These data suggest that SCLC cell lines possess both intrinsic and acquired resistance mechanisms to LSD1 inhibitors.

\section{Neuroendocrine and mesenchymal expression signatures stratify sensitivity to LSD1 inhibition}

Across the SCLC cell line panel, 12 out of 29 tested SCLC cell lines showed greater than $50 \%$ growth inhibition in response to $0.3 \mu \mathrm{M}$ GSK690 treatment at day 17 (Fig. 2A). A non-enzymatic mechanism of action has recently been proposed to account for the anti-tumor activity of LSD1 inhibitors in SCLC [15]. In our cell panel we identified that all SCLC cell lines sensitive to GSK690 express SNAG domain proteins INSM1 or GFI1B, however we also observed examples of INSM1 or GFI1B-expressing cell lines that were also resistant to the drug (Fig. 2C). Thus, expression of SNAG-domain proteins alone is insufficient to define cell lines that will respond to GSK690.

To identify additional biomarkers that predict sensitivity to GSK690 we explored both mutational and gene expression features of SCLC cell lines from available CCLE data [24] LSD1 expression level alone did not correlate with drug sensitivity (Fig. 2B, 2C). Additionally, using both univariate and multivariate association methods, no mutation significantly correlated with cell line sensitivity (data not shown). Analysis of the cell line gene expression by principal component analysis suggests that cell lines sensitive to GSK690 stratify in a group with distinct transcriptional state compared to insensitive cell lines with exception of $\mathrm{NCl}-\mathrm{H} 526$ and $\mathrm{NCl}-\mathrm{H} 1963$ cells that appear to be outliers (Figure S5A). SCLC cell lines can be stratified by neuroendocrine/epithelial (NE) and mesenchymal-like (ML) features. Using established gene expression markers of these SCLC cell states $[16,17]$ we found enrichment in GSK690 
sensitive cell lines in expression of epithelial genes, MYCL and EPCAM, and neuroendocrine genes, GRP, DDC, ASCL1 (Fig. 2B-C, S4). SCLC cell lines resistant to GSK690 featured higher expression of mesenchymal genes MYC, ZEB1, and VIM (Fig. 2B, 2C, S4). These data suggest that neuroendocrine-like SCLC cell lines maybe more sensitive to LSD1 inhibition while mesenchymal-like SCLC states may confer resistance to LSD1 inhibitors.

To experimentally address the intrinsic drug resistance of mesenchymal-shifted SCLC cells to LSD1 inhibitors, we treated a mesenchymal variant form of NCl-H69 cells, NCl-H69V [43] with GSK690. NCl-H69 cells grow in suspension and show enrichment in NE transcription factors FOXA2, SOX2, LEF1, NCAM, Ecadherin (Fig. 2D). In contrast, NCl-H69V cells grow as adherent cells and express a ML signature enriched transcription factors SMAD3, MYC, ZEB1, VIM [19] (Fig. 2D). In line with our hypothesis, mesenchymal-shifted NCl-H69V appeared to be resistant to GSK690 treatment, showing only 22\% growth inhibition compared to $85 \%$ growth inhibition observed in parental $\mathrm{NCl}-\mathrm{H} 69$ cells (Fig. 2D).

To further refine the gene expression signature associated with LSD1 inhibitor resistance, we performed differential expression analysis between LSD1 inhibition sensitive and resistant SCLC cells from CCLE cell line data. Using this approach, we identified a differential gene expression signature (FDR $<5 \%$ and FC > 2) stratifying cell lines sensitive and resistant to LSD1 inhibitors (Fig. 3A). By performing Gene Set Enrichment Analysis (GSEA) [27] on these differential genes, we found that gene sets of ASCL1 targets, epithelial differentiation, and ZEB1 repressive sites are up-regulated in the sensitive cells, whereas EMT, TGF beta and MYC pathway gene sets are significantly up-regulated in the resistant cells (Fig. 3B).

Since sensitivity to LSD1 inhibition was associated with presence or absence of expression of canonical neuroendocrine and mesenchymal markers in our previous analysis, we compared the GSK690 differentially expressed gene signature to an established gene co-expression network that delineates tumor heterogeneity observed in SCLC tumor samples as Neuroendocrine (NE) and Mesenchymal-Like $(M L)[18,19]$. Aligning the LSD1 inhibition differential expression signature to genes defining the NE $(n=$ $1102)$ or $M L(n=2663)$ co-expression networks [19], we found that sensitive cell lines significantly upregulated NE network genes, while resistant lines significantly up-regulated ML network genes (Fig. 3C). In addition, genes up-regulated in LSD1 inhibitor sensitive cell lines significantly overlapped with NE network genes while genes up-regulated in LSD1 inhibitor resistant cell lines significantly overlapped with the ML network genes. (Figure S5B). Overall, these data highlight the context specificity of GSK690 activity in neuroendocrine SCLC subtypes and explain the heterogenous drug responses observed in SCLC cell lines.

We next assessed the potential of the LSD1 sensitivity signature in identification of SCLC patients that might be sensitive or resistant to LSD1 inhibition. Using the differential gene expression signatures that stratify cell lines sensitive and resistant to LSD1 inhibitors (Fig. 3A), TCGA 2015 UCologne SCLC patient dataset was analyzed. Patients with high expression of NE genes correlated with genes increased in expression in the LSD1 sensitivity signature score. Conversely, patients enriched in ML signature overlapped with LSD1 inhibitor resistance gene expression (Fig. 3D). Thus the LSD1 inhibitor gene 
expression signature defined distinct subsets of SCLC patients that are predicted to be either sensitive or resistant to LSD1 inhibitors.

\section{Drug tolerant cells with mesenchymal-like states emerge after LSD1 inhibitor treatment}

SCLC cell lines grow in floating cell aggregates however a subset of cell lines will grow attached to tissue culture plastic $[44,45]$. Cell lines which grow in suspension tend to express neuroendocrine markers while adherently growing cell lines are enriched in mesenchymal biomarkers [19]. SCLC cells that persist after day 10 of $0.3 \mu \mathrm{M}$ GSK690 treatment show profound morphologic changes. $\mathrm{NCl}-\mathrm{H} 526$ cells changed from loose floating aggregates to tightly adherent spheres (Fig. 4A). COR-L88 cells changed morphologically and gained adherence to tissue culture treated plates at day 12 of GSK690 treatment (Fig. 4A).

Since morphological changes are known to occur in SCLC cells that transition to a mesenchymal state [46], we assessed if LSD1 inhibitor persister populations shift to a mesenchymal differentiation state. We performed bulk RNA-seq on 4 sensitive cell lines (NCl-H889, NCl-H1417, NCl-H69 and COR-L88) and 2 insensitive cell lines ( $\mathrm{NCl}-\mathrm{H} 82$ and $\mathrm{NCl}-\mathrm{H} 1694)$. At day 10 of GSK690 treatment, a larger number of genes show changes in all four sensitive cell lines, with the majority of genes showing up-regulation ( $F D R<0.05$, fold change > = 2) (Fig. 4B). Interestingly, only 5 genes and 43 genes were significantly altered after 10 days of GSK690 treatment in 2 GSK690 insensitive models, NCl-H82 and NCl-H1694, respectively (Fig. 4B). Evaluation of common transcriptional changes occurring in sensitive models revealed only seven genes as consistently altered (FDR $<0.05$, fold change $>=2$ ) in expression at day 10 in all four cells lines (Fig. 4C). However, the data showed more convergence at a pathway level with several common pathways identified showing consistent changes due to GSK690 treatment in the four sensitive cell lines. Pathways associated with genes up-regulated by GSK690 treatment are enrichment in epithelialmesenchymal transition pathway, TGF beta signaling, NOTCH signaling as well as focal adhesion related signaling consistent with the observed changes in adhesion (Fig. 4D, S5C). Conversely, pathways enriched in genes down-regulated by GSK690 treatment did not show overlap between different cell lines and include various signaling pathways (Fig. 4D, S5C).

Changes in neuroendocrine and mesenchymal markers were also observed at the protein level in GSK690 sensitive cell models (Figure S6). COR-L88 cells showed the strongest shift into a mesenchymal-like state showing down-regulation of neuroendocrine proteins NSE, GRP, NCAM, and CHGA and neuroendocrine transcription factors FOXA2, ASCL1, and SOX2. COR-L88 cells also showed strong up-regulation of the mesenchymal protein VIM. Other GSK690 sensitive cell models showed only small changes in protein levels across our panel with GRP reduction and ZEB1 and $\mathrm{CDH} 2$ up-regulation being the most consistent markers of response (Figure S6). We observed no consistent changes in the protein levels of any neuroendocrine and mesenchymal markers in GSK690 insensitive cell lines. Importantly, the extent of mesenchymal gene induction in GSK690 treated SCLC cells does appear comparatively lower relative to what we observed in mesenchymal-shifted NCl-H69V cells and thus may represent a 'partial EMT' [47]. 


\section{LSD1 inhibitor persister cells evolve from epigenetically distinct subpopulations}

To define the origin of mesenchymal-like persister cells, we conducted single cell RNA-seq analysis on $\mathrm{NCl}-\mathrm{H} 69$ cells treated with DMSO or $0.3 \mu \mathrm{M}$ GSK690 for 21 days. Harmony [31] was applied to integrate DMSO and treatment groups so that gene expression within each single cell subpopulation can be directly compared to obtain subpopulation specific differentially expressed (DE) genes after treatment (Fig. 5A). To identify distinct cell subpopulations, unsupervised cell clustering was performed and five clusters were selected manually after visualization using uniform manifold approximation and projection (UMAP) (Fig. 5B and S7A). Cell subpopulations in Cluster 0 and 3 were increased in $0.3 \mu \mathrm{M}$ GSK690 treated group relative to the DMSO group (Figure S7A). In contrast, cell subpopulations in Cluster 1 and 2 in DMSO group were reduced after 21 days of LSD1 inhibitor treatment (Figure S7A). There was no significant difference in the Cluster 4 cell subpopulation between DMSO and treated groups. Cluster 2 was identified as a neuroendocrine-like subpopulation, as neuroendocrine transcriptional factors GRP and ASCL1 were specifically up-regulated in this subpopulation (Fig. 5B, S8A). Both GRP and ASCL1 expression were strongly reduced in Cluster 2 after treatment (Figure S7B). In addition, the mean expression of neuroendocrine markers (NE score) including ASCL1, CHGA, GRP, INSM1, NCAM1, FOXA2 and SOX2 were significantly reduced in Cluster 2 after treatment (Fig. 5C, left), suggesting cells in this cluster are losing NE features. On the other hand, the mean expression of 76 EMT marker genes from the Broad MSigDB Hallmark Signatures (EMT score) was up-regulated in multiple cell clusters after treatment, but most significantly increased in Cluster 0 (Fig. 5C, right). This observation is supported by the significant up-regulation of EMT and lung cancer survival pathways in Cluster 0 after treatment (Figure S8B). Altogether, these results highlight the loss of NE cell subpopulation, and the emergence of several transcriptionally distinct, mesenchymal-like populations in $\mathrm{NCl}-\mathrm{H} 69$ cells resistant to LSD1 inhibitors.

In an attempt to assess the reversibility of the mesenchymal shift observed in LSD1 inhibitor persister cells we performed drug washout experiments. After 14 days of GSK690 treatment in $\mathrm{NCl}-\mathrm{H} 526$ and $\mathrm{NCl}-$ $\mathrm{H} 69$ cells, anti-proliferative effects occurred concomitant with the up-regulation of mesenchymal markers CDH2, SNA1, MYC, VIM, and ZEB1 and the down-regulation of neuroendocrine markers GRP, ASCL1, and CHGA (Figure S9). After 7 days of drug washout, gene expression signatures recovered in both cell lines to levels similar to that observed in DMSO controls. Moreover, after 7 days of drug wash-out, cells regained sensitivity to LSD1 inhibition to a similar extent as untreated cell lines (Figure S9). These data suggest that the mesenchymal shift in SCLC cells treated with LSD1 inhibitors is reversible and caused by an "epi-stable" mesenchymal-like differentiation state.

LSD1 inhibitor resistance occurs through chromatin accessibility changes for genes associated with neuroendocrine and mesenchymal like programs

We next sought to define the extent of epigenetic reprogramming in LSD1 inhibitor drug tolerant cells by employing an assay for transposase-accessible chromatin using sequencing (ATAC-seq). ATAC-seq data 
in $\mathrm{NCl}-\mathrm{H} 69$ cells treated with $0.3 \mu \mathrm{M}$ GSK690 for 21 days was highly reproducible between biological replicates and showed clear enrichment at specific genomic regions (Figure S10A). Consistent with observed changes in gene expression, differentially open or closed chromatin accessibility peaks were primarily identified at distal regulatory regions of genes after 21 days of GSK690 treatment compared to controls (Fig. 6A, S10B). GREAT gene ontology analysis [39] for genes acquiring open chromatin accessibility peaks near regulatory regions suggests enrichment in pathways involved in mesenchyme morphogenesis and regulation of epithelial cell differentiation (Fig. 6B). In contrast, genes that acquire closed chromatin do not show any significant pathway enrichment.

To study the transcription factor programs regulating drug resistant SCLC cells, we identified the transcription factor motifs represented in differentially accessible, closed or open, chromatin regions after GSK690 treatment. We used ChromVar [40], a package designed for inferring transcription factor activity from ATAC-seq based on chromatin accessibility levels at TF binding motifs genome-wide (Fig. 6C). Our analysis demonstrated that NEUROD1/NEUROD2 and ASCL1 DNA binding motifs were significantly closed following GSK690 treatment. The DNA binding motif for TEAD4 is the only significantly opened region with the treatment. TEAD4 is a downstream mediator of YAP1 activity, which has been previously identified as a possible phenotypic modulator in a subset of non-neuroendocrine SCLC cell lines [48]. These data indicate that mesenchymal-like drug tolerance in SCLC cell lines occurs through epigenetic reprogramming mediated through changes in transcription factor programs and chromatin accessibility changes that alter gene expression.

\section{Discussion}

In this study we have identified common features of intrinsic and acquired drug resistance to LSD1 inhibitors through epigenetic changes in SCLC neuroendocrine transcriptional programs. Epigenetic plasticity is known to contribute to the poor durability of responses of SCLC to current therapies as selection of mesenchymal enriched clones become enriched in refractory disease found in relapse [49, 50]. Many SCLC cell lines analyzed in this study show either no response or only partial response to LSD1 inhibitors even after 21 days of treatment, suggesting many SCLC cell lines have either a pre-existing drug resistant population or are capable of transitioning to a drug resistant state. Experimentally neuroendocrine SCLC cell lines can transition from neuroendocrine into mesenchymal phenotypes when treated with targeted or chemotherapies $[19,44,45,49]$. This observation is clinically relevant as SCLC tumors are often composed of epigenetically heterogeneous cells with either a neuroendocrine or a mesenchymal profile $[18,19,49]$.

Despite early literature suggesting LSD1 inhibitors would be broadly active in numerous cancers including colon, prostate and breast cancer $[12,51,52]$ the generation of more selective LSD1 inhibitors has clarified the extent of LSD1 inhibitor activity primarily in AML and SCLC $[5,53]$. One possible explanation for this discrepancy is suggested from the recent reports identifying non-catalytic mechanisms of action of current LSD1 inhibitors being explored in the clinic. Inhibition of the interaction of LSD1 with SNAG domain-containing proteins INSM1 or GFI1B impacts expression of key 
neuroendocrine lineage transcriptional regulators in SCLC including ASCL1 [15]. Our data demonstrates that all cell lines sensitive to LSD1 inhibitors express either INSM1 or GFI1B, however a subset of cell lines resistant to the drug also express these genes. Thus, expression of these SNAG domain-containing proteins alone does not predict sensitivity to LSD1 inhibitors. Interestingly, LSD1 has been found to interact with the SNAG domain of SNAIL and implicated in the transcriptional repression of epithelial markers during EMT transition in breast cancer cells [54]. The role of LSD1 in the SNAG domain of targeted proteins appears to be context and lineage specific.

Our results demonstrate that SCLC cells which have evolved non-neuroendocrine, mesenchymal-like transcriptional programs are resistant to LSD1 inhibition. These findings are consistent with Mohammad et al., who identified TGF-beta pathway signatures negatively correlate with LSD1 inhibitor sensitivity in SCLC [5]. The TGF-beta pathway is known to regulate EMT pathways in cancer as well as in normal development [46]. In cell lines sensitive to LSD1 inhibitors we did observe enrichment for TGF beta pathway genes as well as genes involved in axonal and EMT pathways after LSD1 inhibitor treatment. Therefore, these data are potentially consistent with our observation that neuroendocrine and mesenchymal differentiation markers stratify sensitivity of SCLC cell lines to LSD1 inhibitors. Augert et. al also identified heterogenous responses to the LSD1 inhibitor ORY-1001 in a screen of SCLC patientderived xenograft (PDX) models [13]. In their study, PDX models capable of NOTCH activation and concomitant ASCL1 downregulation were enriched in response to LSD1 inhibition. In gene expression studies following GSK690 treatment we did observe induction of NOTCH pathway genes in COR-L88 and $\mathrm{NCl}-\mathrm{H} 1417$ cells but not to the same extent in $\mathrm{NCl}-\mathrm{H} 69$ or $\mathrm{NCl}-\mathrm{H} 889$ cells. This suggests that NOTCH pathway independent mechanisms of LSD1 inhibitor response exist in SCLC.

This study highlights the remarkable plasticity of SCLC to evolve into drug resistant mesenchymal-like states. Along these lines, we found expression of the neuroendocrine transcription factor ASCL1 was down-regulated after GSK690 treatment in most models with the exception of $\mathrm{NCl}-\mathrm{H} 526$ cells which shows a very low expression level of ASCL1 and is reported that the interaction between LSD1 and SNAG domain protein GFI1B plays a key role in this cell line [15].The extent of mesenchymal gene induction in GSK690 treated SCLC cells does appear comparatively lower relative to what we observed in mesenchymal-shifted NCl-H69V cells and thus may represent a 'partial EMT' [47]. In support of this model, drug wash out experiments suggest these changes are "epistable" as cells revert to neuroendocrine phenotypes within 7 days of drug removal. Additionally, we found many SCLC cell lines analyzed in this study show only partial response to LSD1 inhibitors even after 21 days of treatment, suggesting many SCLC cell lines are capable of transitioning to a drug resistant state. Single cell RNA-seq suggests that this resistant population likely represents an acquired drug tolerant state represented by transcriptional reprogramming that is maintained under drug selection. Consistent with this finding, ATAC-seq data in NCl-H69 cells highlighted a shift in the utilization of transcription factors from ASCL1 to TEAD4 in drug resistant cells. Interestingly, expression of TEAD4 and downstream YAP and TAZ have recently been highlighted to represent a unique subtype of non-ASCL1 driven SCLC [48]. 
A recent phase I study of GSK2879552 in relapsed or refractory SCLC found a disease control rate of $14 \%$ (four of 29 patients) [55]. High rates of adverse events, including thrombocytopenia and encephalopathy, lead to the termination of the study. Our data suggests that only a segment of SCLC patients, with a defined neuroendocrine differentiation state, will likely benefit from LSD1 inhibitors. In LSD1 inhibitor treated patients, a mesenchymal-like resistant population is likely to persist. Thus, emphasis should focus on drug combination approaches that can sustain clinical responses by eliminating cells that maintain residual disease. Experimentally, it has been demonstrated that SCLC cells treated with chemotherapy [49] or radiotherapy [56] obtain mesenchymal characteristics that confer drug resistance. Thus, the efficacy of LSD1 inhibitors in SCLC patients heavily pre-treated with chemotherapy will be an important consideration. The recent disclosures of additional LSD1 inhibitors, IMG-7289, INCB059872, CC-90011, under clinical development for SCLC highlights the importance of clarifying the mechanism of action as well as further defining the clinical application of these drugs [6].

\section{Conclusions}

We discovered that epigenetic plasticity contributes to heterogeneous responsiveness of SCLC to LSD1 inhibitors. Sensitivity to LSD1 inhibitors in SCLC is confined primarily to cells that express neuroendocrine transcriptional programs. Selection of a TEAD4-driven mesenchymal-like subpopulation is likely to present a barrier to effective single-agent responses in the clinic. Drug combinations specifically targeting intrinsic and adaptive mesenchymal-like resistant populations to sustain effective clinical response will be of high interest for future clinical development.

\section{Abbreviations}

ASCL1: Achaete-scute homolog 1

ATAC-seq: Assay for Transposase-Accessible Chromatin using sequencing

ATCC: American Type Culture Collection

BRD4: Bromodomain-containing protein 4

CCLE: Broad Institute Cancer Cell Line Encyclopedia

CREBBP: cAMP-response element-binding protein-binding protein

GESA: gene set enrichment analysis

EMT: Epithelial-mesenchymal transition

EP300: Histone acetyltransferase p300 or E1A-associated protein p300

EZH2: Enhancer of zeste homolog 2 
GFI1B: Growth Factor Independent 1B

GRP: Gastrin-releasing peptide

INSM1: Insulinoma-associated protein 1

LSD1/KDM1A: Lysine-specific histone demethylase 1A

MLL1/2: Mixed lineage leukemia protein 1/2

NEUROD1: Neurogenic differentiation 1

PDX: Patient-derived xenografts

RB1: retinoblastoma protein 1

SCLC: small cell lung cancer

scRNA-seq: single cell RNA sequencing

SNAG: SNAIL/Gfi-1 Family Zinc Finger Proteins

TEAD4: TEA domain family member 4

VIM: vimentin

ZEB1: Zinc finger E-box-binding homeobox 1

\section{Declarations}

Ethics approval and consent to participate

Not applicable.

Consent for publication

All authors consent for publications.

Availability of data and materials

All data generated and/or analyzed during this study are available within this paper and from the corresponding authors.

Competing interests

W.Y., C-Y.C, S.S., T.X., M.O., T.N., J.F., T.A.P. are employed by and hold stock in Pfizer, Inc. No potential conflicts of interests were disclosed by the other authors. 


\section{Funding}

All sources of funding for the research reported here are supported by Pfizer, Inc.

\section{Authors' contributions}

W.Y. designed and executed experiments; collected, analyzed, and interpreted data; and contributed to writing and editing the manuscript. C-Y.C. performed bioinformatic analyses; analyzed and interpreted data; and contributed to writing the manuscript. T.X. performed bioinformatic analyses. M.O. executed experiments and analyzed data. T.C.N., and J.F. executed and analyzed histopathology. A.R.U. reviewed and provided input on the contents of the manuscript. S.S. designed the study, analyzed and interpreted data; contributed to writing the manuscript. T.A.P. designed the study and wrote the manuscript. All authors reviewed and approved the final manuscript.

\section{Acknowledgements}

The authors thank Jill Hallin, Ying Ding, Mike Greig for their efforts in the early phase of this project. We also thank Elizabeth McMillan for reviewing and providing input to the manuscript. We are grateful to Robert Hollingsworth for the support and review of the manuscript.

\section{Authors' information}

Affiliations

Oncology Research Discovery, Pfizer Worldwide Research and Development, 10777 Science Center Dr, San Diego, CA 92121, USA

Wen Yan, Chi-Yeh Chung, Tao Xie, Mark Ozeck, Timothy C. Nichols, Jessica Frey, Shikhar Sharma, Thomas A. Paul

Arcus Biosciences, 3928 Point Eden Way, Hayward, CA 94545, USA Akshata R. Udyavar

Corresponding authors

Thomas A. Paul, Phone: 858-526-4241; E-mail: thomas.a.paul@pfizer.com;

\section{References}

1. George, J., et al., Comprehensive genomic profiles of small cell lung cancer. Nature, 2015. 524(7563): p. 47-53.

2. Otterson, G.A., et al., Phase II study of the histone deacetylase inhibitor Romidepsin in relapsed small cell lung cancer (Cancer and Leukemia Group B 30304). J Thorac Oncol, 2010. 5(10): p. 1644-8.

3. Lenhart, R., et al., Sensitivity of Small Cell Lung Cancer to BET Inhibition Is Mediated by Regulation of ASCL 1 Gene Expression. Mol Cancer Ther, 2015. 14(10): p. 2167-74. 
4. Poirier, J.T., et al., DNA methylation in small cell lung cancer defines distinct disease subtypes and correlates with high expression of EZH2. Oncogene, 2015. 34(48): p. 5869-78.

5. Mohammad, H.P., et al., A DNA Hypomethylation Signature Predicts Antitumor Activity of LSD1 Inhibitors in SCLC. Cancer Cell, 2015. 28(1): p. 57-69.

6. Fang, Y., G. Liao, and B. Yu, LSD1/KDM1A inhibitors in clinical trials: advances and prospects. J Hematol Oncol, 2019. 12(1): p. 129.

7. Hakimi, M.A., et al., A core-BRAF35 complex containing histone deacetylase mediates repression of neuronal-specific genes. Proc Natl Acad Sci U S A, 2002. 99(11): p. 7420-5.

8. Ceballos-Chavez, M., et al., Control of neuronal differentiation by sumoylation of BRAF35, a subunit of the LSD1-CoREST histone demethylase complex. Proc Natl Acad Sci U S A, 2012. 109(21): p. 808590.

9. Iwase, S., et al., Characterization of BHC80 in BRAF-HDAC complex, involved in neuron-specific gene repression. Biochem Biophys Res Commun, 2004. 322(2): p. 601-8.

10. Shi, Y., et al., Histone demethylation mediated by the nuclear amine oxidase homolog LSD1. Cell, 2004. 119(7): p. 941-53.

11. Laurent, B., et al., A specific LSD1/KDM1A isoform regulates neuronal differentiation through H3K9 demethylation. Mol Cell, 2015. 57(6): p. 957-70.

12. Metzger, E., et al., LSD1 demethylates repressive histone marks to promote androgen-receptordependent transcription. Nature, 2005. 437(7057): p. 436-9.

13. Augert, A., et al., Targeting NOTCH activation in small cell lung cancer through LSD1 inhibition. Sci Signal, 2019. 12(567).

14. Vinyard, M.E., et al., CRISPR-suppressor scanning reveals a nonenzymatic role of LSD1 in AML. Nat Chem Biol, 2019. 15(5): p. 529-539.

15. Takagi, S., et al., LSD1 Inhibitor T-3775440 Inhibits SCLC Cell Proliferation by Disrupting LSD1 Interactions with SNAG Domain Proteins INSM1 and GFI1B. Cancer Res, 2017. 77(17): p. 4652-4662.

16. Rudin, C.M., et al., Molecular subtypes of small cell lung cancer: a synthesis of human and mouse model data. Nat Rev Cancer, 2019. 19(5): p. 289-297.

17. Rudin, C.M., et al., Author Correction: Molecular subtypes of small cell lung cancer: a synthesis of human and mouse model data. Nat Rev Cancer, 2019. 19(7): p. 415.

18. Wooten, D.J., et al., Systems-level network modeling of Small Cell Lung Cancer subtypes identifies master regulators and destabilizers. PLoS Comput Biol, 2019. 15(10): p. e1007343.

19. Udyavar, A.R., et al., Novel Hybrid Phenotype Revealed in Small Cell Lung Cancer by a Transcription Factor Network Model That Can Explain Tumor Heterogeneity. Cancer Res, 2017. 77(5): p. 10631074.

20. Dhanak, D., Drugging the cancer epigenome. Proceedings of the 104th annual meeting of the american association for cancer research, 2013. 
21. Guibourt, N.O., A.; Laria, J.P.C. , Phenylcyclopropylamine derivatives and their medical use. PCT Int. Appl., WO 2010084160 A1 201007292010.

22. Li, B. and C.N. Dewey, RSEM: accurate transcript quantification from RNA-Seq data with or without a reference genome. BMC Bioinformatics, 2011. 12: p. 323.

23. Anders, S. and W. Huber, Differential expression analysis for sequence count data. Genome Biol, 2010. 11(10): p. R106.

24. Barretina, J., et al., The Cancer Cell Line Encyclopedia enables predictive modelling of anticancer drug sensitivity. Nature, 2012. 483(7391): p. 603-7.

25. Ritchie, M.E., et al., limma powers differential expression analyses for RNA-sequencing and microarray studies. Nucleic Acids Res, 2015. 43(7): p. e47.

26. Gu, Z., R. Eils, and M. Schlesner, Complex heatmaps reveal patterns and correlations in multidimensional genomic data. Bioinformatics, 2016. 32(18): p. 2847-9.

27. Subramanian, A., et al., Gene set enrichment analysis: a knowledge-based approach for interpreting genome-wide expression profiles. Proc Natl Acad Sci U S A, 2005. 102(43): p. 15545-50.

28. Langfelder, P. and S. Horvath, WGCNA: an R package for weighted correlation network analysis. BMC Bioinformatics, 2008. 9: p. 559.

29. Stuart, T., et al., Comprehensive Integration of Single-Cell Data. Cell, 2019. 177(7): p. 1888-1902 e21.

30. Hafemeister, C. and R. Satija, Normalization and variance stabilization of single-cell RNA-seq data using regularized negative binomial regression. Genome Biol, 2019. 20(1): p. 296.

31. Korsunsky, l., et al., Fast, sensitive and accurate integration of single-cell data with Harmony. Nat Methods, 2019. 16(12): p. 1289-1296.

32. Becht, E., et al., Dimensionality reduction for visualizing single-cell data using UMAP. Nat Biotechnol, 2018.

33. Langmead, B. and S.L. Salzberg, Fast gapped-read alignment with Bowtie 2. Nat Methods, 2012. 9(4): p. 357-9.

34. Zhang, Y., et al., Model-based analysis of ChIP-Seq (MACS). Genome Biol, 2008. 9(9): p. R137.

35. Kent, W.J., et al., BigWig and BigBed: enabling browsing of large distributed datasets. Bioinformatics, 2010. 26(17): p. 2204-7.

36. Robinson, J.T., et al., Integrative genomics viewer. Nat Biotechnol, 2011. 29(1): p. 24-6.

37. Ramirez, F., et al., deepTools: a flexible platform for exploring deep-sequencing data. Nucleic Acids Res, 2014. 42(Web Server issue): p. W187-91.

38. Love, M.I., W. Huber, and S. Anders, Moderated estimation of fold change and dispersion for RNA-seq data with DESeq2. Genome Biol, 2014. 15(12): p. 550.

39. McLean, C.Y., et al., GREAT improves functional interpretation of cis-regulatory regions. Nat Biotechnol, 2010. 28(5): p. 495-501.

40. Schep, A.N., et al., chromVAR: inferring transcription-factor-associated accessibility from single-cell epigenomic data. Nat Methods, 2017. 14(10): p. 975-978. 
41. Morgan, M.A. and A. Shilatifard, Chromatin signatures of cancer. Genes Dev, 2015. 29(3): p. 238-49.

42. Clinical Lung Cancer Genome, P. and M. Network Genomic, A genomics-based classification of human lung tumors. Sci Transl Med, 2013. 5(209): p. 209 ra153.

43. Khan, M.Z., et al., Identification and characterisation in vitro of cells with a non-SCLC cell-like phenotype derived from a continuous SCLC cell line. Anticancer Res, 1991. 11(5): p. 1687-95.

44. Carney, D.N., et al., Establishment and identification of small cell lung cancer cell lines having classic and variant features. Cancer Res, 1985. 45(6): p. 2913-23.

45. Gazdar, A.F., et al., Characterization of variant subclasses of cell lines derived from small cell lung cancer having distinctive biochemical, morphological, and growth properties. Cancer Res, 1985. 45(6): p. 2924-30.

46. Xu, J., S. Lamouille, and R. Derynck, TGF-beta-induced epithelial to mesenchymal transition. Cell Res, 2009. 19(2): p. 156-72.

47. Jolly, M.K., et al., Implications of the Hybrid Epithelial/Mesenchymal Phenotype in Metastasis. Front Oncol, 2015. 5: p. 155.

48. Horie, M., et al., YAP and TAZ modulate cell phenotype in a subset of small cell lung cancer. Cancer Sci, 2016. 107(12): p. 1755-1766.

49. Krohn, A., et al., Tumor cell heterogeneity in Small Cell Lung Cancer (SCLC): phenotypical and functional differences associated with Epithelial-Mesenchymal Transition (EMT) and DNA methylation changes. PLoS One, 2014. 9(6): p. e100249.

50. Brambilla, E., et al., Cytotoxic chemotherapy induces cell differentiation in small-cell lung carcinoma. J Clin Oncol, 1991. 9(1): p. 50-61.

51. Huang, Y., et al., Novel oligoamine analogues inhibit lysine-specific demethylase 1 and induce reexpression of epigenetically silenced genes. Clin Cancer Res, 2009. 15(23): p. 7217-28.

52. Lim, S., et al., Lysine-specific demethylase 1 (LSD1) is highly expressed in ER-negative breast cancers and a biomarker predicting aggressive biology. Carcinogenesis, 2010. 31(3): p. 512-20.

53. McGrath, J.P., et al., Pharmacological Inhibition of the Histone Lysine Demethylase KDM1A Suppresses the Growth of Multiple Acute Myeloid Leukemia Subtypes. Cancer Res, 2016. 76(7): p. 1975-88.

54. Lin, T., et al., Requirement of the histone demethylase LSD1 in Snai1-mediated transcriptional repression during epithelial-mesenchymal transition. Oncogene, 2010. 29(35): p. 4896-904.

55. Bauer, T.M., et al., Phase I, Open-Label, Dose-Escalation Study of the Safety, Pharmacokinetics, Pharmacodynamics, and Efficacy of GSK2879552 in Relapsed/Refractory SCLC. J Thorac Oncol, 2019. 14(10): p. 1828-1838.

56. Meredith, S.L., et al., Irradiation Decreases the Neuroendocrine Biomarker Pro-Opiomelanocortin in Small Cell Lung Cancer Cells In Vitro and In Vivo. PLoS One, 2016. 11(2): p. e0148404.

\section{Figures}


A.

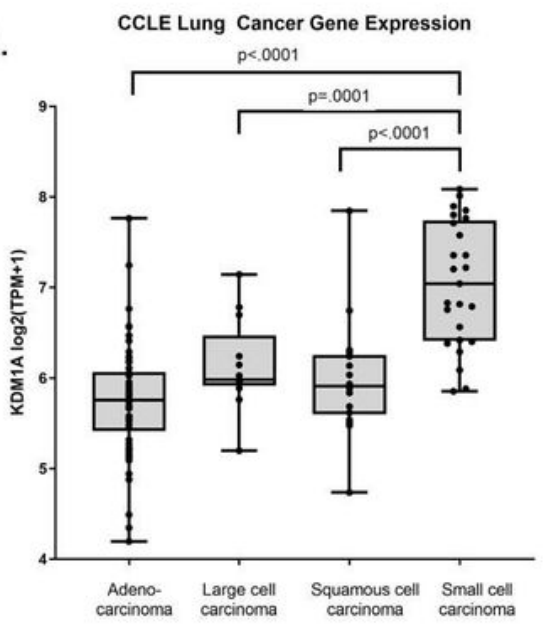

B.
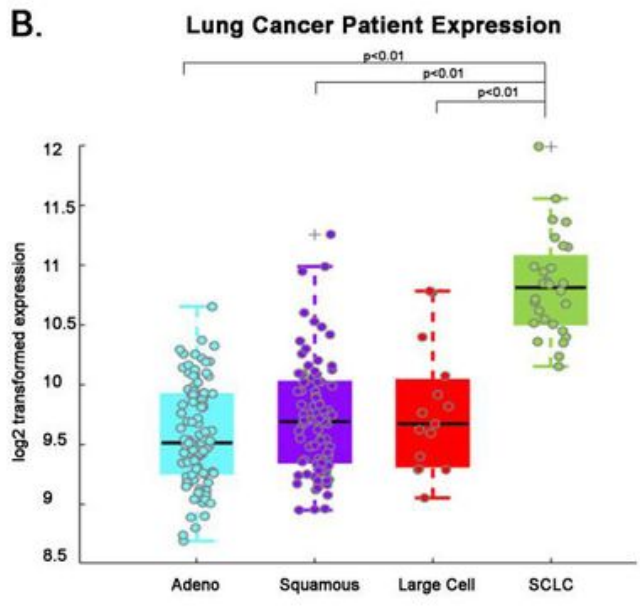

C.

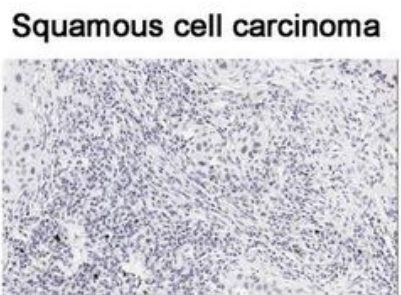

Adenocarcinoma
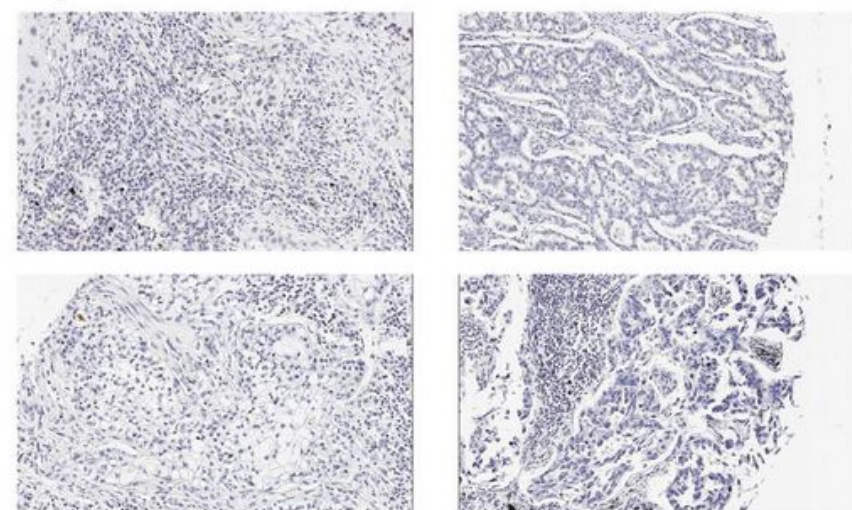

$p=0.022$

D.

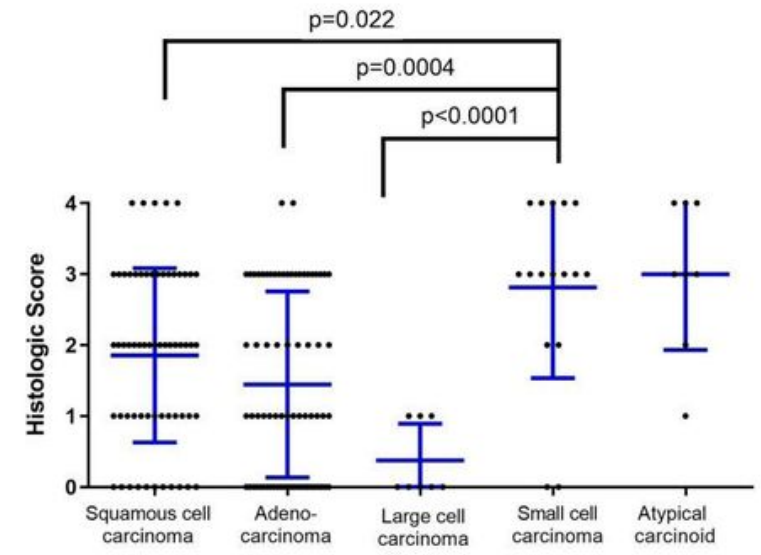

\section{Figure 1}

LSD1 is highly expressed in small cell lung cancer relative to other lung cancer subtypes. (A) Expression of LSD1 (KDM1A) mRNA from lung cancer cell lines derived from the CCLE dataset at the Broad Institute. (B) LSD1 expression data in 261 primary lung tumors from Clinical Lung Cancer Genome Project (CLCGP). (C) Representative images of LSD1 expression by immunohistochemistry (IHC) in lung cancer tissue microarray. (D) Quantitation of LSD1 expression in 200 microarray dot tissue samples all from a 
broad array of human lung cancer histologic subtypes and including 20 normal lung samples for comparison. LSD1 IHC immunoreactivity was given a histologic semi-quantitative score (0-4; 4 being highest intensity staining) for each sample. Data presented represent mean $+/-1$ standard deviation (SD). Statistical significance was determined by one-way ANOVA with Tukey HSD post-hoc test for (A), (B) and (D).
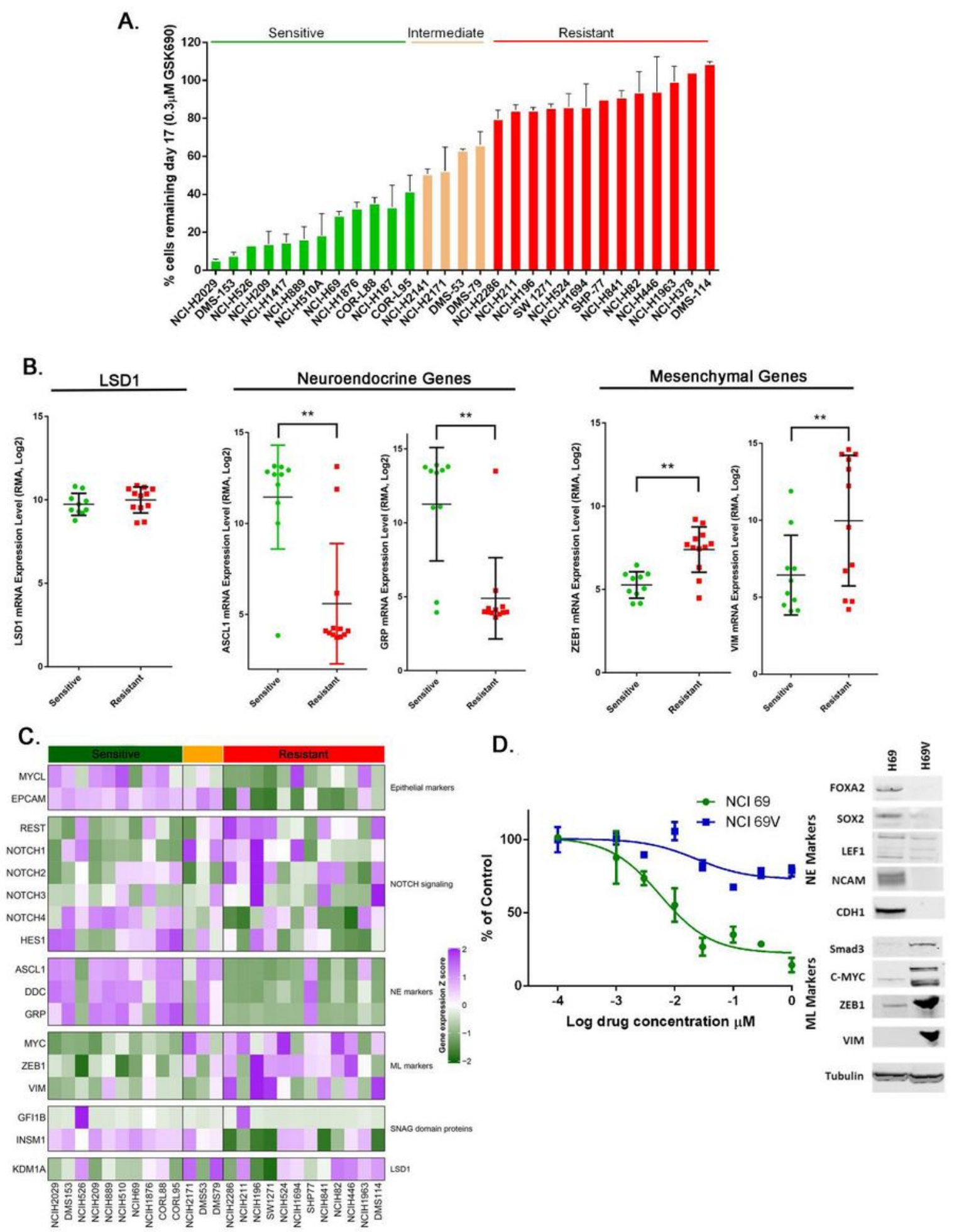

Figure 2 
Differential sensitivity of SCLC cell lines to LSD1 inhibitors stratifies with expression of neuroendocrine markers. (A) SCLC cell sensitivity to $0.3 \mu \mathrm{M}$ GSK690 at day 17 based on the percentage of remaining cells relative to DMSO control. Long-term proliferation assays were run in 3 technical replicates. Sensitive cell lines are reflected by greater than $50 \%$ growth inhibition, resistant cell lines at less than $20 \%$ growth inhibition. (B) Expression of GRP, ASCL1, VIM, and ZEB1 in GSK690 sensitive and resistant cell lines based of CCLE mRNA expression data. Two sample t-test p-values $<0.05$ are shown. (C) Heatmap of CCLE mRNA expression of specific marker genes defining SCLC subtypes. Gene expression values are shown as row normalized z scores from log2(TPM+1). (D) GSK690 dose response in NCl-H69 compared to $\mathrm{NCl}-\mathrm{H} 69 \mathrm{~V}$ cells at day 17 . Western blot using indicated antibodies demonstrating protein expression of neuroendocrine (NE) and mesenchymal (ML) markers in $\mathrm{NCl}-\mathrm{H} 69$ and $\mathrm{NCl}-\mathrm{H} 69 \mathrm{~V}$ cells. 
A.

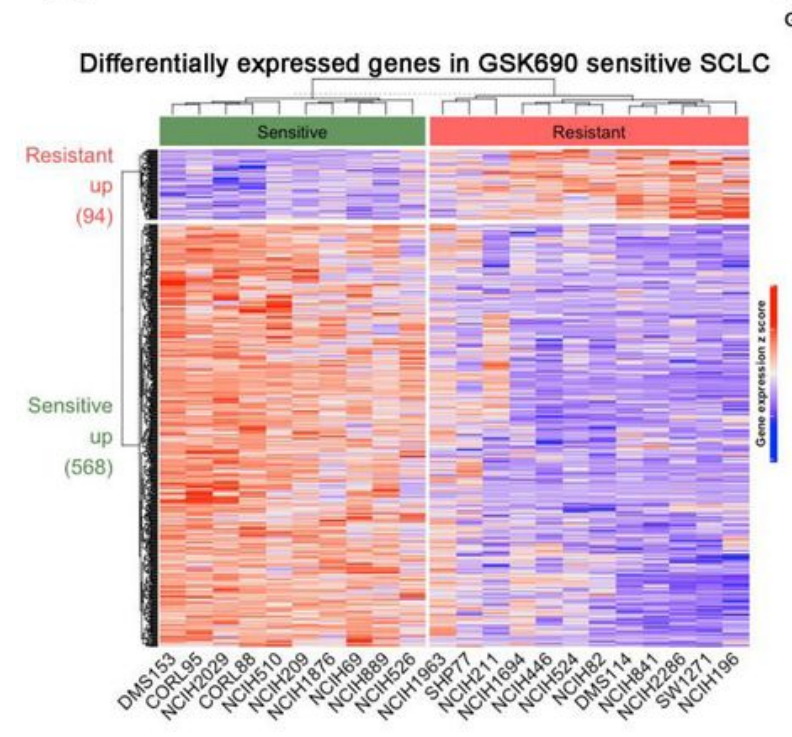

C.
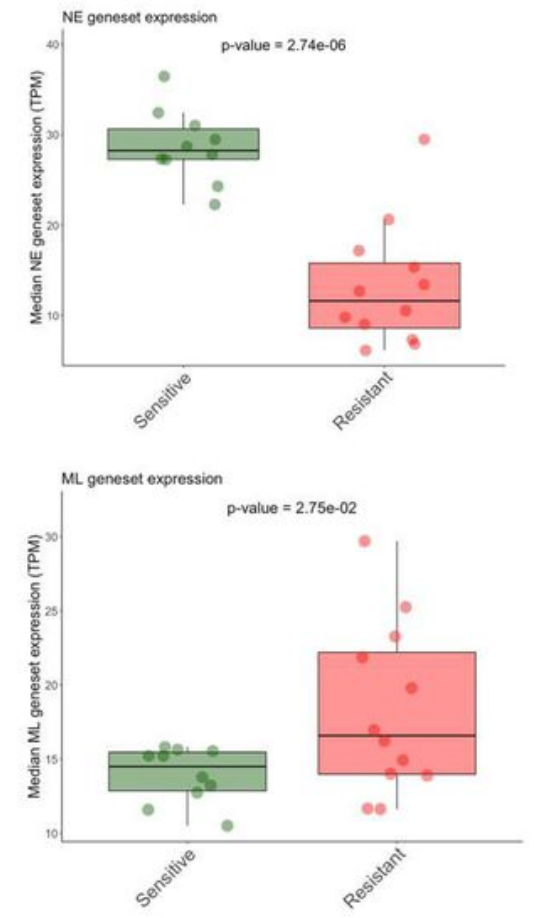

D.
B.
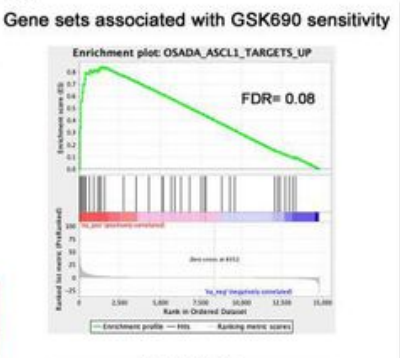

Gene sets associated with GSK690 resistanc
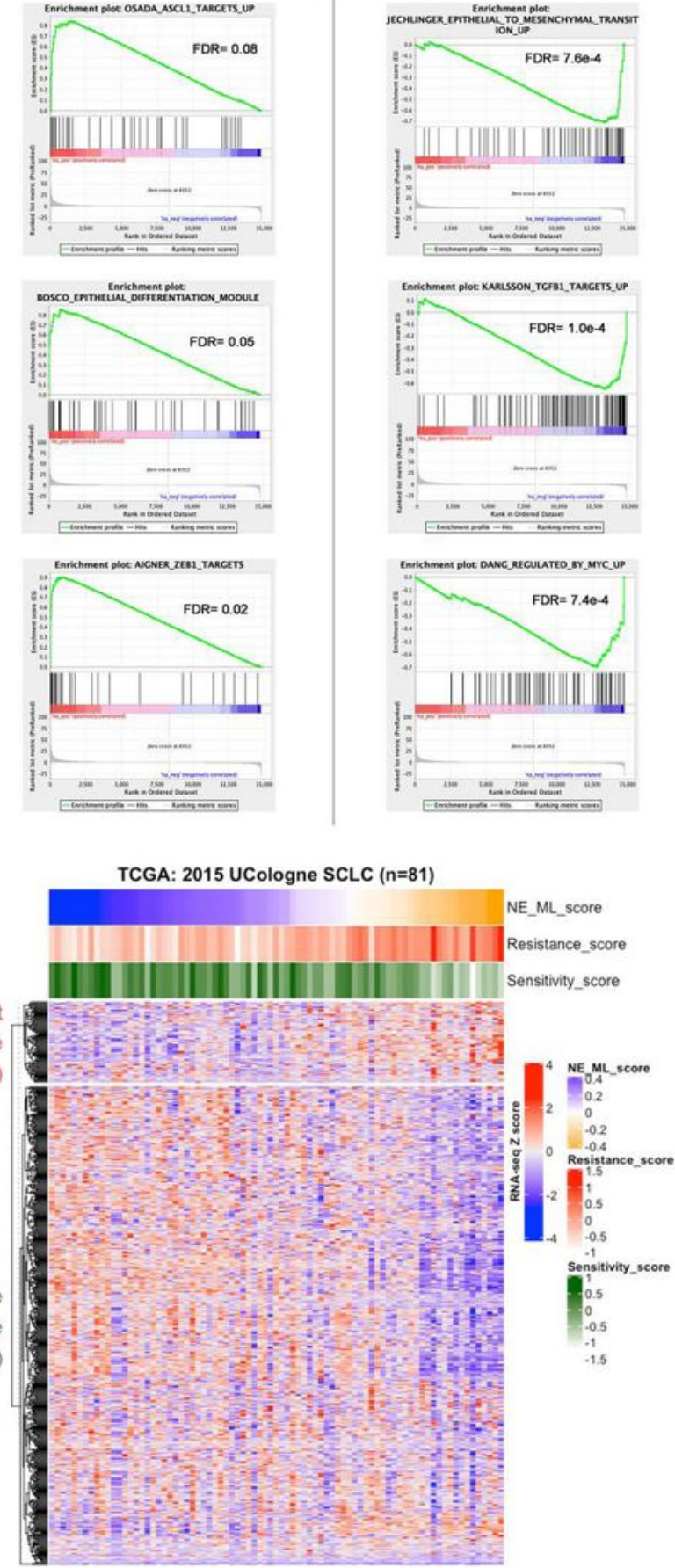

\section{Figure 3}

Connection of LSD1 sensitivity signature with neuroendocrine and mesenchymal-like network signatures. (A) CCLE gene expression heatmap with hierarchical clustering of differentially expressed (DE) genes between sensitive $(n=10)$ and resistant $(n=12)$ cell lines. Gene expression values are shown as row zscore. DE gene cutoff is FDR $<5 \%$ and FC > 2. (B) Gene Set Enrichment Analysis (GSEA) of differential gene expression between sensitive and resistant cell lines from (A). (C) Boxplot depicting the CCLE 
median expression values (TPM) of previously published Neuroendocrine (NE) and Mesenchymal-like $(\mathrm{ML})$ signatures [19] in sensitive and resistant cell lines from (A). Two sample t-test $p$-values are shown. (D) Heatmap representation of RNA-seq Z score for TCGA 2015 UCologne SCLC data. The column represents 81 SCLC patient tumors, and the row represents LSD1i signature genes generated from $(A)$. Genes captured for each signature from the TCGA samples were as follows (captured genes/total signature genes): $3426 / 3471$ for NE signature; $1169 / 1179$ for ML signature; $533 / 568$ for LSD1i sensitivity signature; $90 / 94$ for resistance signature. The NE-ML score was calculated by subtracting the NE score from the ML score for each tumor. Hypergeometric test p-values are shown.

A.

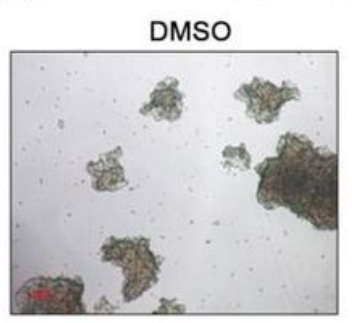

$\mathrm{NCl}-\mathrm{H} 526$ (day 10)

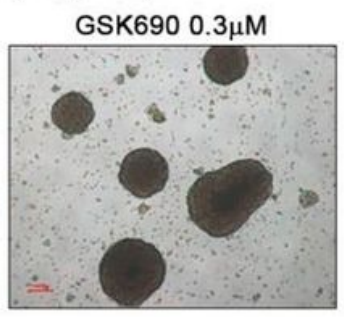

COR-L88 (day 12)
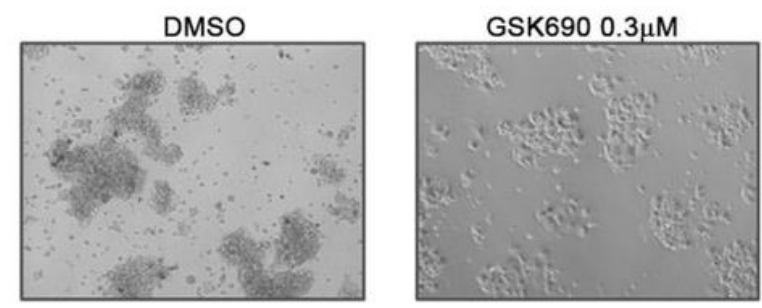

C.

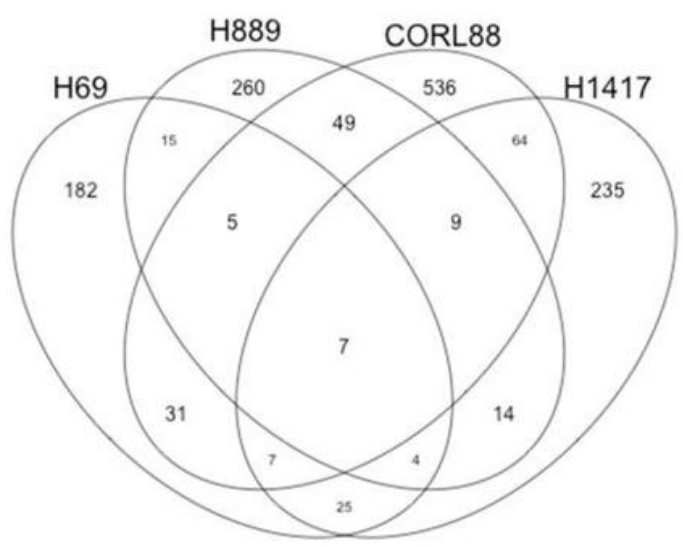

B.

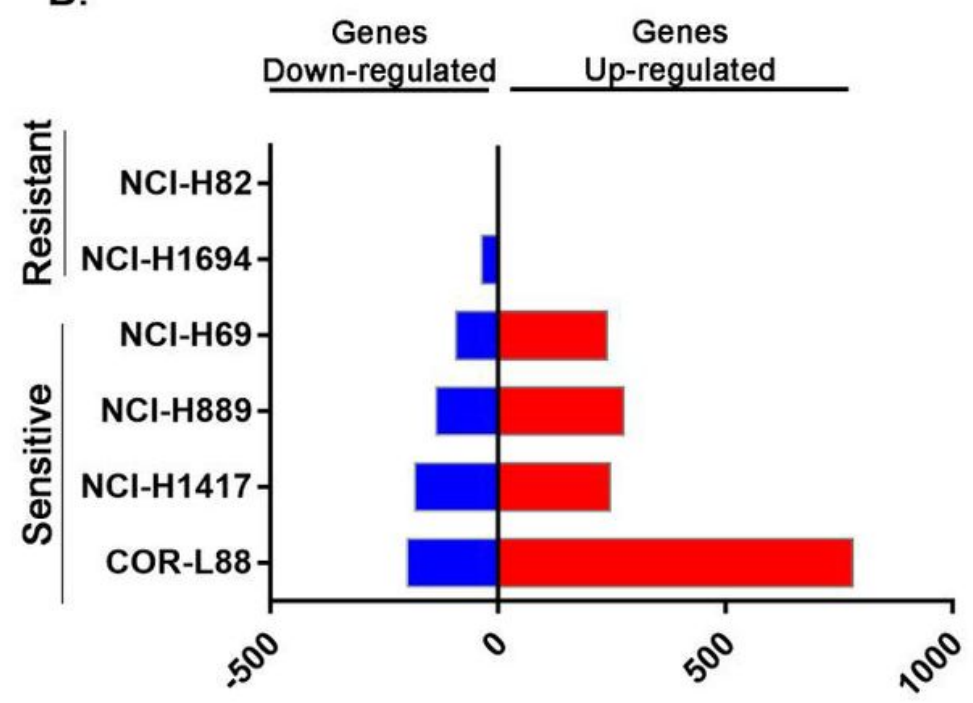

D. Pathways changed by GSK690 treatment

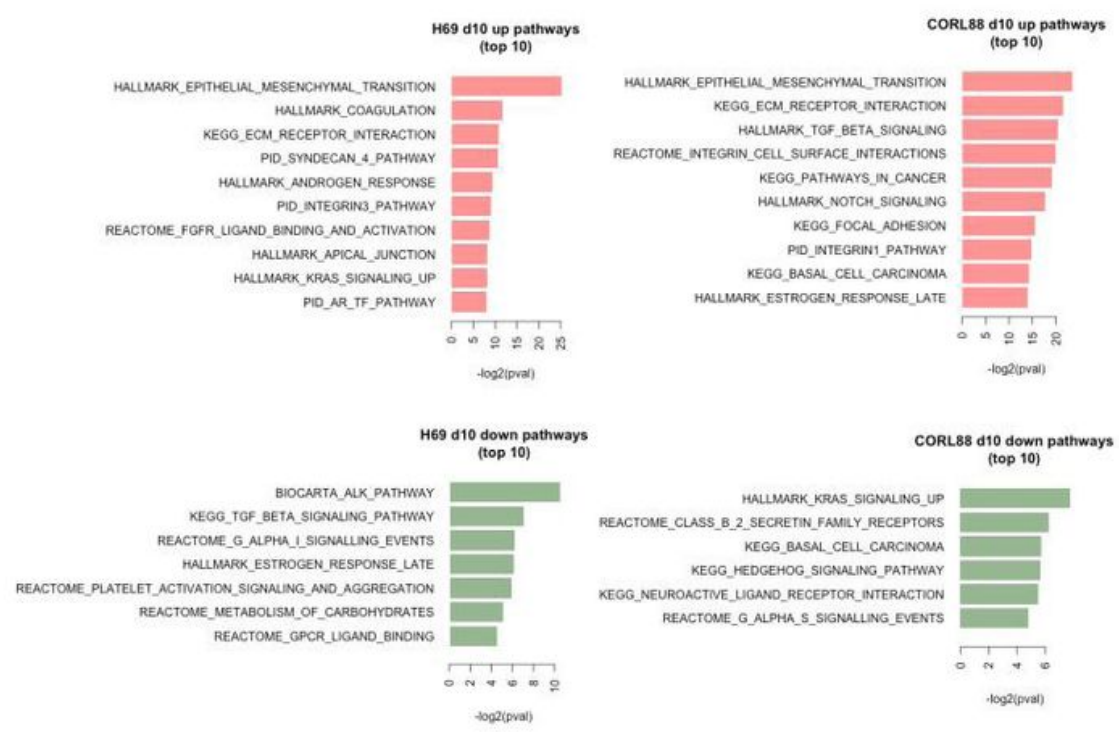




\section{Figure 4}

LSD1 inhibitor treatment results in gene expression changes in axonal and EMT pathways (A) Microscope images of NCl-H526 and COR-L88 cell clusters grown in tissue culture following treatment with DMSO or $0.3 \mu \mathrm{M}$ GSK690 for indicated times. (B) RNA-seq analysis of genes significantly altered (FDR < 0.05, fold change >=2) in expression in GSK690 sensitive cell lines $\mathrm{NCl}-\mathrm{H} 69, \mathrm{NCl}-\mathrm{H} 1417, \mathrm{NCl}-\mathrm{H} 889$, and COR-L88 and GSK690 insensitive cell lines NCl-H82 and NCl-H1694 treated with vehicle (DMSO) or $0.3 \mu \mathrm{M}$ GSK690 for 10 days. (C) Venn diagrams reflecting overlap of genes significantly up-regulated or down-regulated by GSK690 treatment between SCLC cell lines. (D) Pathway analysis of genes altered in expression (FDR $<0.05$, fold change $>=2$ ) by GSK690 treatment utilizing MSigDB pathway databases. Top 10 enriched pathways are shown with the p-value cutoff of 0.05 . 


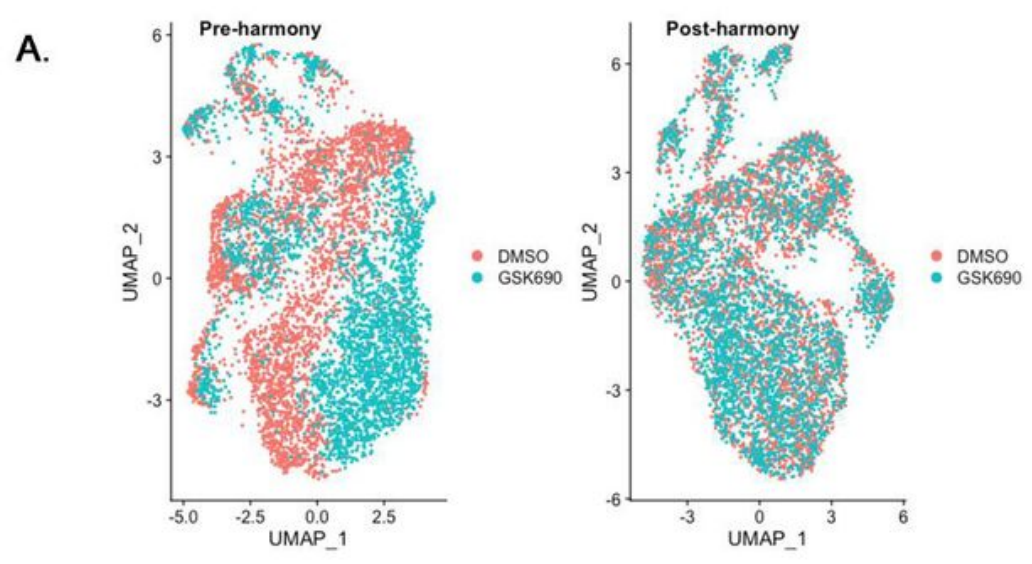

B.
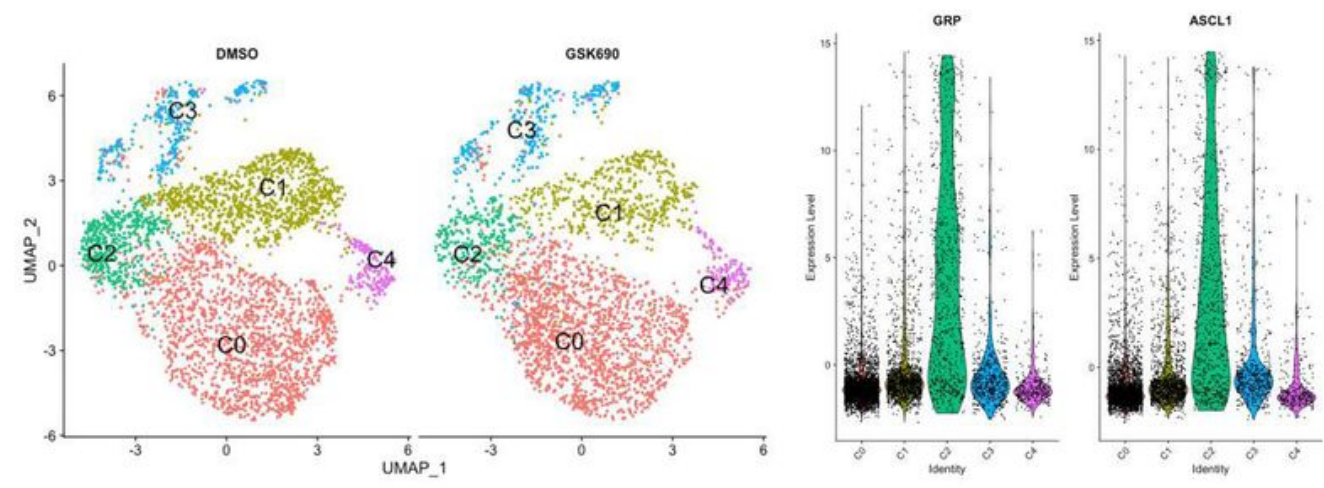

C.
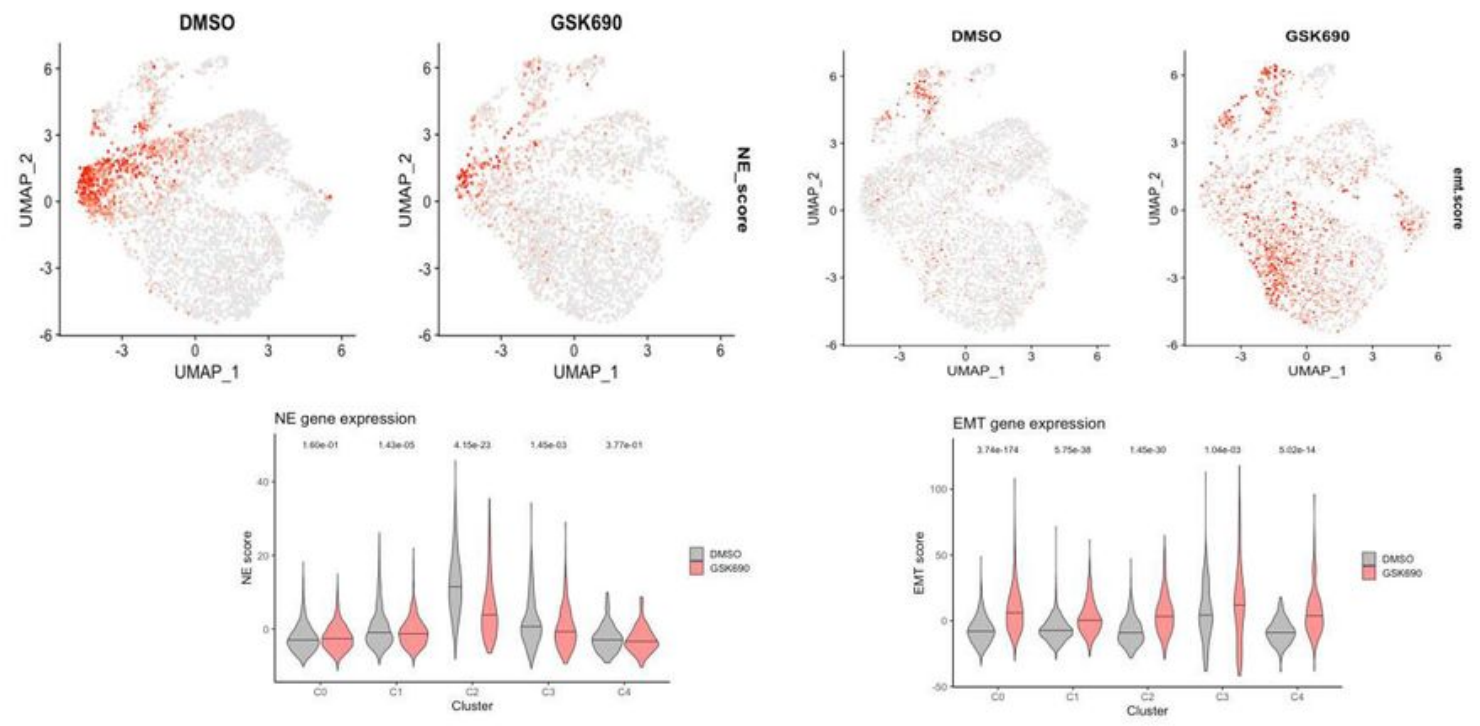

\section{Figure 5}

Single Cell RNA-seq reveals emergence of cell subpopulations enriched in EMT pathways following LSD1 inhibitor treatment (A) Uniform Manifold Approximation and Projection (UMAP) representation of pre- and post-Harmony integrated single cell RNA-seq data following treatment with vehicle (DMSO) or $0.3 \mu \mathrm{M}$ GSK690 for 21 days. (B) UMAP representation of cell clustering and GRP and ASCL1 expression violin 
plot in each cluster. (C) Mean expression of NE or ML gene sets in UMAP or as violin plots in each cluster. Two sample t-test p-values are shown.

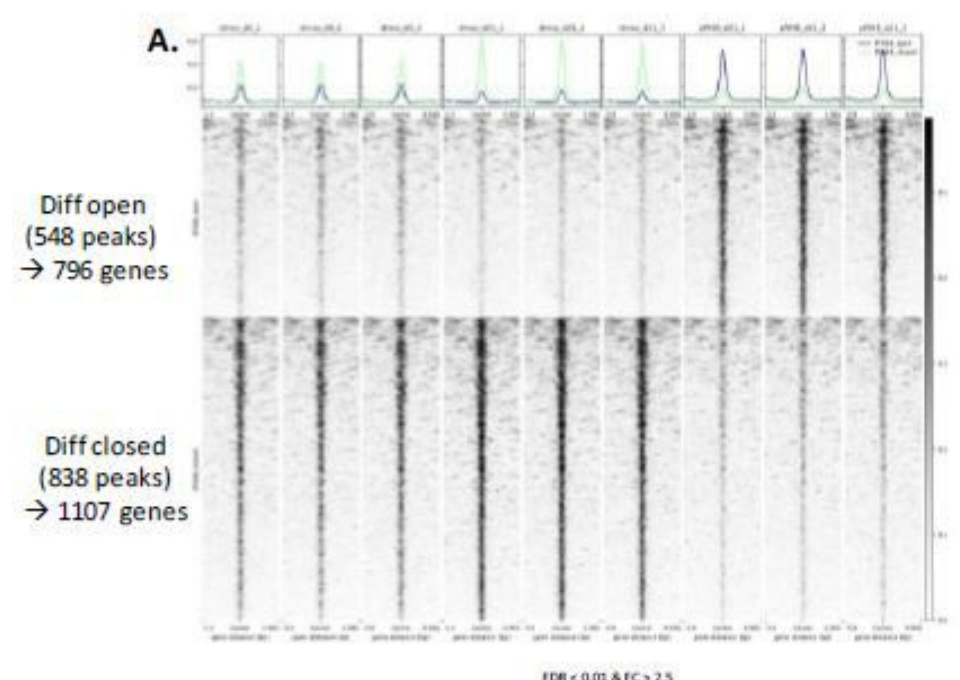

B.

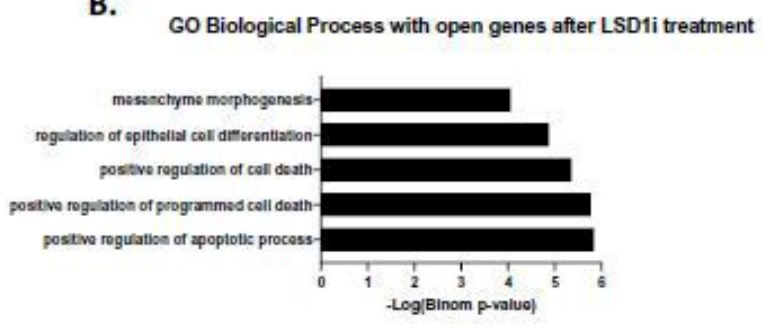

C.
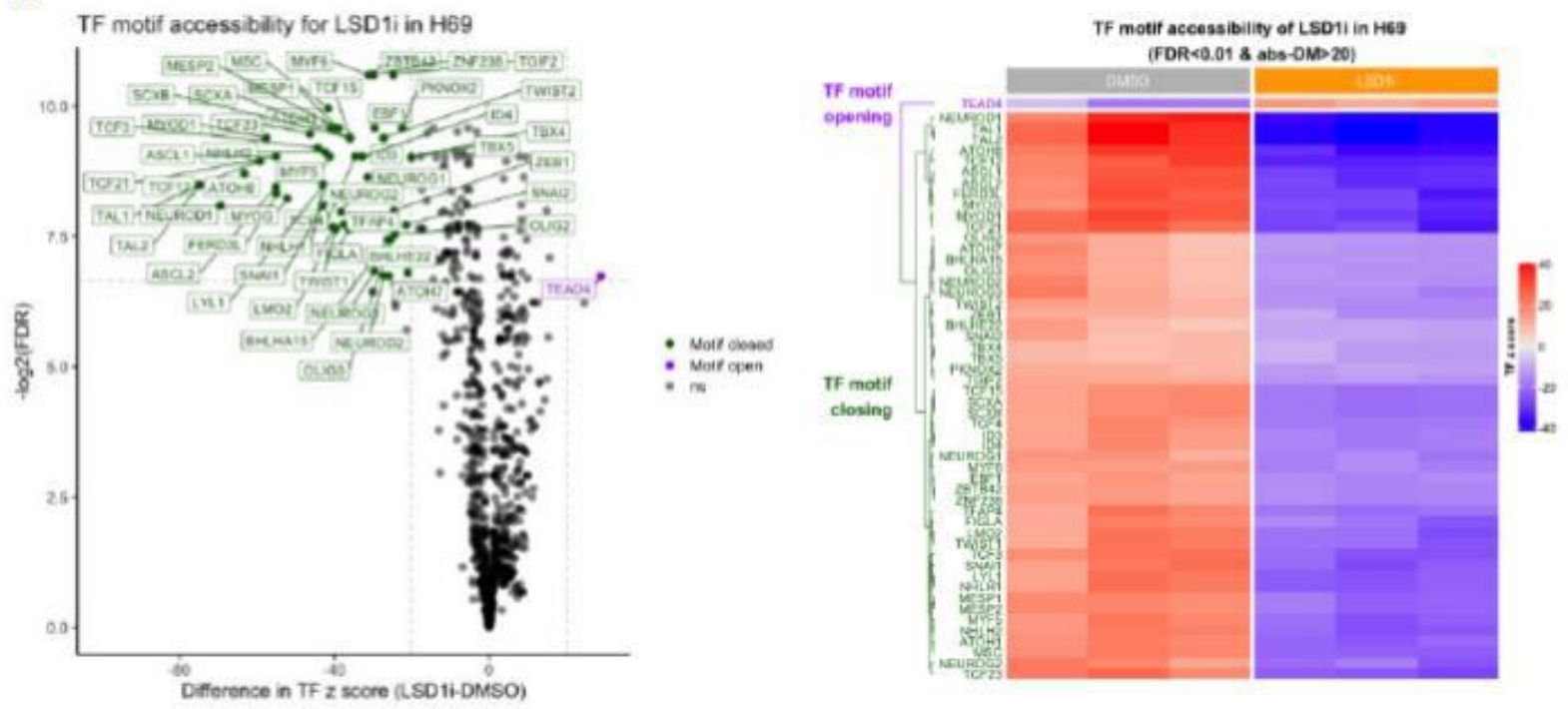

Figure 6

Chromatin accessibility changes for genes associated with neuroendocrine and mesenchymal expression signatures (A) ATAC-seq signal at differentially open or closed regions (FDR $<1 \%$ and fold change $>2.5$ ) between vehicle (DMSO day 0 and day 21) or $0.3 \mu \mathrm{M}$ GSK690n (day 21) in NCl-H69 cells ( $n=3$ ). (B) GO 
Biological Process using GREAT analysis with open genes associated with $0.3 \mu \mathrm{M}$ GSK690 treatment. (C) Volcano plot (left) showing the significantly open or closed TF DNA binding motifs (FDR $<1 \%$ and difference in mean z-score > 20) with $0.3 \mu \mathrm{M}$ GSK690 using chromVar analysis. Heatmap (right) representing TF $z$ scores of significantly open or closed TF DNA binding motifs $(n=3)$.

\section{Supplementary Files}

This is a list of supplementary files associated with this preprint. Click to download.

- Yanetal.Additionalfile102152021.pdf 